Textures and Microstructures, Vol. 33, pp. 207-217 Reprints available directly from the publisher Photocopying permitted by license only
(C) 1999 OPA (Overseas Publishers Association) N.V. Published by license under the Gordon and Breach Science Publishers imprint. Printed in Malaysia.

\title{
DETERMINATION OF RESIDUAL STRESSES IN WCu GRADIENT MATERIALS
}

\author{
G. BOKUCHAVA ${ }^{\mathrm{a}, *}$, N. SHAMSUTDINOV ${ }^{\mathrm{a}}$, \\ J. SCHREIBER ${ }^{b}$ and M. STALDER ${ }^{b}$ \\ ${ }^{a}$ Frank Laboratory of Neutron Physics, Joint Institute for \\ Nuclear Research, 141980 Dubna, Moscow Region, Russia; \\ ${ }^{\mathrm{b}}$ Fraunhofer Institut für zerstörungsfreie Prüfverfahren, \\ Außenstelle Dresden, Krügerstraße 22, D-01326 Dresden, Germany
}

Residual stress measurements were carried out on copper-tungsten gradient materials by means of neutron diffraction on the High Resolution Fourier Diffractometer on IBR-2, JINR, Dubna. The samples so far investigated had a concentration profile that approximates the gradient by step functions. The results show an averaged positive stress (stretch) in the copper phase and negative (compression) in the tungsten phase. A comparison to analytical linear elastic calculations shows that the measured values are nearly an order of magnitude too low, whereas however, a qualitative agreement of the stress distribution exists. That this quantitative discrepancy can be attributed to the model's simplifying assumption of pure elastic behaviour can be shown by recent calculations that also include plasticity and show a much better agreement with the experimental values. Single peak evaluation also strongly suggests that plasticity does indeed play a significant role.

Keywords: Strain measurement; Reverse time-of-flight; Residual stress;

WCu gradient material

\section{INTRODUCTION}

Modern technology sometimes requires components with properties that are not met in one material alone. Therefore one tries to combine materials with different properties. On their interface, however, significant residual stresses may occur that finally give rise to cracking

\footnotetext{
* Corresponding author.
} 
or failure of the component. Therefore a comparatively new approach is to vary the materials not abruptly but continuously thus decreasing residual stress discontinuities; see for example Rabin and Shiota (1995); Ilschner and Cherradi (1995).

For example, a promising material system for future fusion reactor walls is a copper-tungsten (WCu) gradient material (Itoh et al., 1996). Copper, an excellent heat conductor, is too soft and not heat resistant. Tungsten, on the other hand, has got the highest melting point of all metals but is a poor heat conductor (see Table I). The different heat expansion coefficients, however, pose a serious problem on the coexistence of the two phases if one keeps in mind that the intended use of the component is such that it will undergo a temperature change in the order of $1000^{\circ} \mathrm{Cduring}$ both manufacturing and operation. Both analytical and Finite Element (FEM) calculations predict that the residual stresses due to this temperature change can be significantly decreased in the gradient material if compared with a two layer sample.

According to the phase diagram both constituents do not mix at all, so that, rather than forming a graded alloy, they exhibit something like a salt and pepper structure with more salt grains on one end and more pepper on the other. WCu gradient materials can be produced by different metallurgical procedures, such as sintering of metal powders, hot isostatic pressing (Joensson, 1996) and electrochemical processing (Jedamzik et al., 1997). In this article we present the results of neutron diffraction measurements of residual stresses in WCu gradient materials and compare them to the available theoretical calculations.

\section{EXPERIMENT}

Several composite pills of different $\mathrm{W} / \mathrm{Cu}$ ratio were studied including both pure tungsten and copper samples in order to determine the stress

TABLE I Some selected properties of the component materials

\begin{tabular}{lrc}
\hline & Copper & Tungsten \\
\hline Heat conductivity $\left(\mathrm{Wm}^{-1} \mathrm{~K}^{-1}\right)$ & 403 & 174 \\
Yield strength $(\mathrm{MPa})$ & 60 & 550 \\
$E$-modulus $(\mathrm{GPa})$ & 145 & 411 \\
Melting point $\left({ }^{\circ} \mathrm{C}\right)$ & 1083 & 3410 \\
Thermal expansion coeff. $(\mathrm{ppm})$ & 16.6 & 4.5 \\
\hline
\end{tabular}


free lattice parameter $a_{0}$. Two gradient samples were prepared at Dresden Technical University by hot isostatic pressing (Joensson, 1996) with a three- and eight-layer structure respectively (Fig. 1). They were cylindrical in shape with a diameter of about $11 \mathrm{~mm}$ and a length of $9 \mathrm{~mm}$.

The neutron diffraction measurements were carried out at HRFD (High Resolution Fourier Diffractometer, Frank Laboratory of Neutron Physics, JINR, Dubna) which has been described in the literature earlier (Aksenov et al., 1996). The used Fourier method (Pöyry et al., 1975; Hiismäki et al., 1988) is also called reverse time-of-flight method (RTOF) and yields like the TOF-method a full diffraction pattern for a fixed scattering geometry so that Rietveld refinement (see Fig. 2(a)) can be applied for evaluation of both phase ratios (see Fig. 2(b)) and lattice spacings taking into account all $h \mathrm{kl}$-reflections. The Rietveld refinement is vastly described in the literature, see for example Young

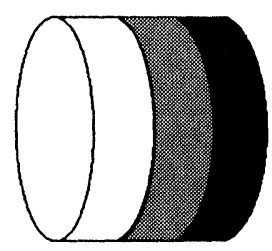

FIGURE 1 Sample geometry.

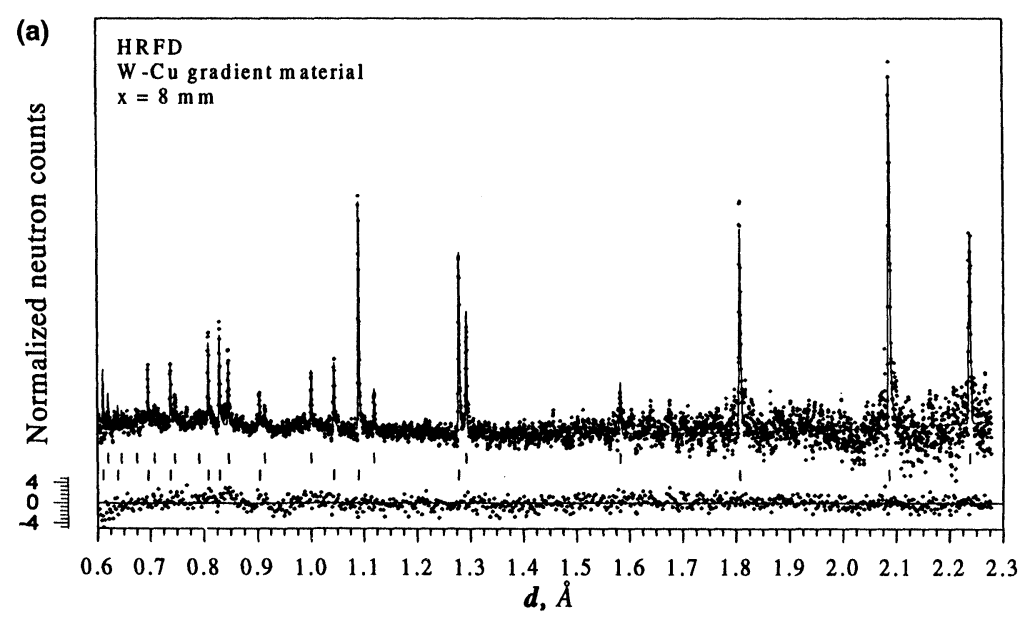

FIGURE 2(a) 


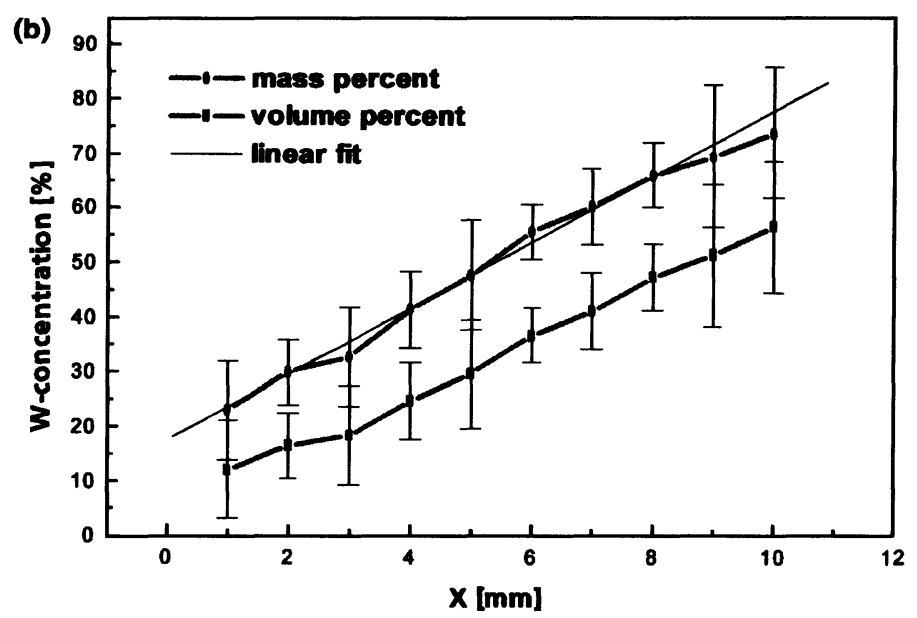

FIGURE 2(b)

FIGURE 2 (a) Diffraction spectrum with Rietveld refinement. (b) Tungsten phase fraction of the eight-layer sample determined by Rietveld refinement.

(1993). Single peak behaviour was only investigated to check elastic anisotropy. The scattering geometry is shown in Fig. 3. The gauge volume was $1 \times \pi \cdot(11 / 2)^{2} \mathrm{~mm}^{3}$. By rotating the sample in the beam by means of a triaxial goniometer and using two detector banks at $76^{\circ}$ and $45^{\circ}$ respectively, lattice distortions can be determined in a sufficient number of spatial directions.

Strains have been calculated from lattice distortions according to the definition:

$$
\varepsilon=\frac{a-a_{0}}{a_{0}}
$$

where $a$ is lattice parameter.

The stresses can be computed by using the following law:

$$
\sigma_{i j}=\left(\frac{E}{1+\nu}\right) \varepsilon_{i j}+\left(\frac{\nu E}{(1+\nu)(1-2 \nu)}\right) \delta_{i j} \varepsilon_{k k} .
$$

$E$ and $\nu$ are the Young's modulus and Poisson's ratio respectively.

With the eight-layered sample we had enough experimental points to run a minimization procedure assuming that there is no averaged stress in axial direction (which is also the gradient direction). So, the stresses in 


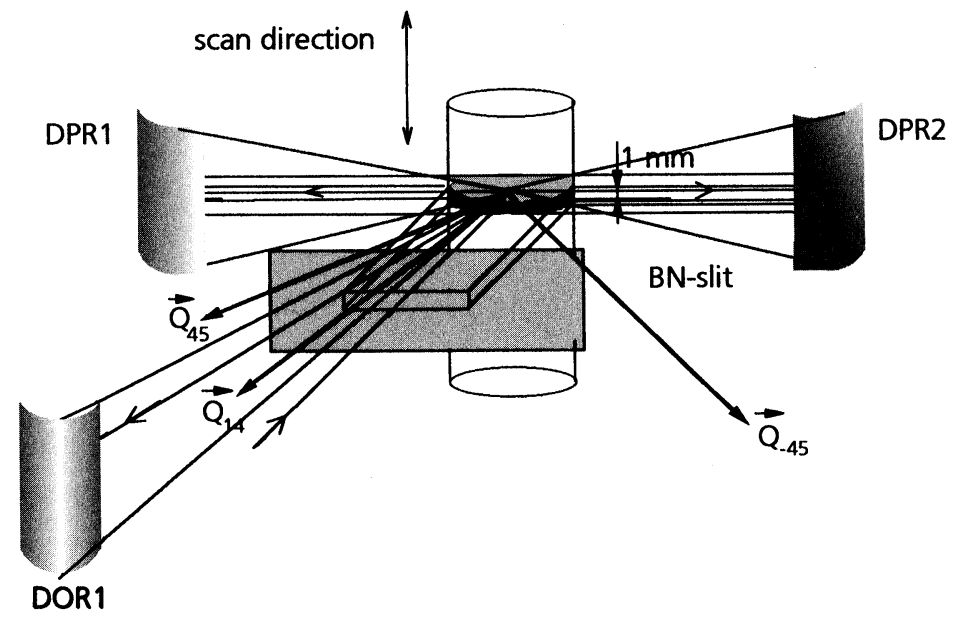

FIGURE 3 Scattering geometry.

this case have been determined without knowledge of the $a_{0}$-value. With the three layered sample we obtained lattice parameters only for two scattering angles. This was sufficient, because the stress state was assumed to have the sample's cylindrical symmetry thus simplifying the stress tensor.

\section{RESULTS AND DISCUSSION}

All samples so far were investigated at room temperature whereas the processing took place at about $1000^{\circ} \mathrm{C}$. Due to the fact that the thermal expansion coefficient of copper exceeds the one of tungsten by a factor of nearly four the copper, upon cooling to room temperature, shrank considerably more than the tungsten. Therefore one would expect a positive strain in the copper phase (pulled apart by the not as much contracting tungsten) whereas the tungsten should exhibit a compressional strain.

Figure 4(a) and (b) displays the theoretical strain and stress of a sample consisting of each a layer of pure copper and pure tungsten after a temperature change of $100^{\circ} \mathrm{C}$ from an assumed stress-free state. It should be kept in mind that even at these comparatively small temperature changes the stress developed has already reached the yield stress for copper, so that elasticity alone will probably not be sufficient to explain the results. Corresponding calculations have also been made for 

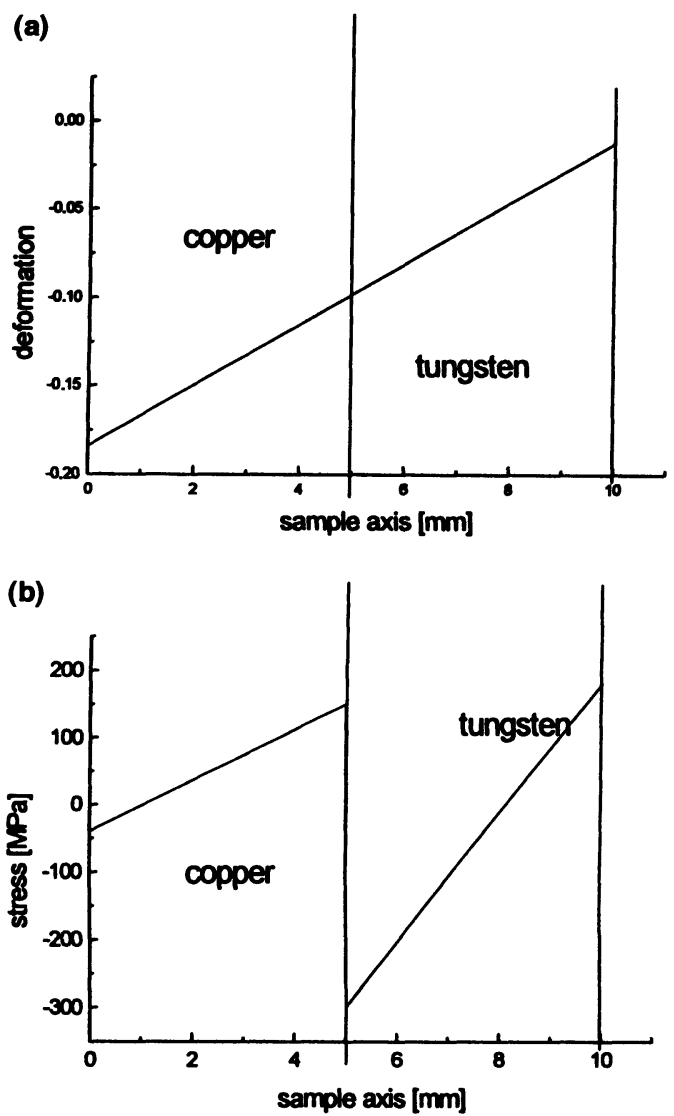

FIGURE 4 Deformation (a) and stress (b) in a two layer system.

a three- and five-layer sample (Fischer, 1996) implying a decrease of residual stresses as the number of layers is increased. Measurements have been carried out at the following samples: single mixture samples, a sample with three layers (Fig. 1) and a sample with eight layers of stepwise varying concentration.

The single mixture samples exhibit the tendency that is also expected from theory: the copper grains are under tension (positive stress), the tungsten grains under compression (negative stress) (Fig. 5). The only values that do not agree with this statement are those for tungsten for the mixture with the lowest tungsten content $(30 \%)$, which have to be taken with a grain of salt, because tungsten is a comparatively bad neutron 
scatterer and at that low concentration does not allow very accurate strain analysis (which is to a certain degree implied by the larger error bars). The reason why the weighted sum of stresses of both phases seems to be slightly out of balance (especially for the 50/50 sample) can be explained by the fact that the $x$-axis depicts mass- $\%$ and not volume- $\%$. Fifty mass- $\%$ tungsten, due to its much larger density, corresponds to only $30 \%$ volume. Weighting the stresses correctly by volume rather than mass the stresses balance much better (Fig. 5).

The three-layer sample was the only one that could be compared with theoretical results. Fig. 6 compares the weighted perpendicular stresses (macrostresses) with the results of an analytical calculation that includes plasticity. The experimentally determined values agree with the model within the margin of error, whereas a linear elastic model (Fischer, 1996) showed a vast overestimation of the real behaviour. This is a well known fact which has been attributed to the negligence of plasticity in models describing WCu materials earlier (Itoh et al., 1996).

Figure 7 shows a plot of the shift of several selected reflections against the factor of anisotropy $\Gamma=\left(h^{2} k^{2}+h^{2} l^{2}+k^{2} l^{2}\right) /\left(h^{2}+k^{2}+l^{2}\right)^{2}$. In the elastic model according to Kroener and Eshelby the relative shift should be a linear function of $\Gamma$. Since this is clearly not the case, the aforementioned hypothesis is reinforced that plastic deformation is part of the game.

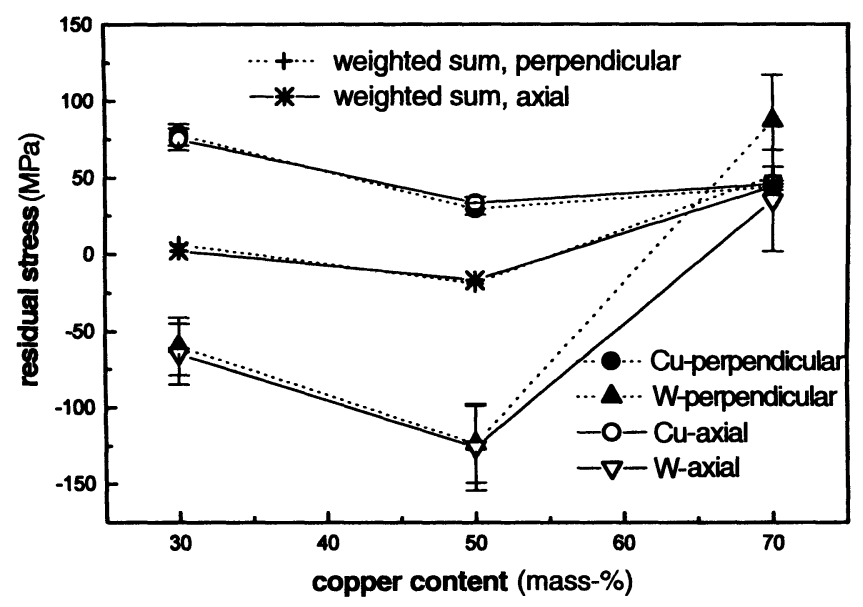

FIGURE 5 Residual load share between the two phases. 


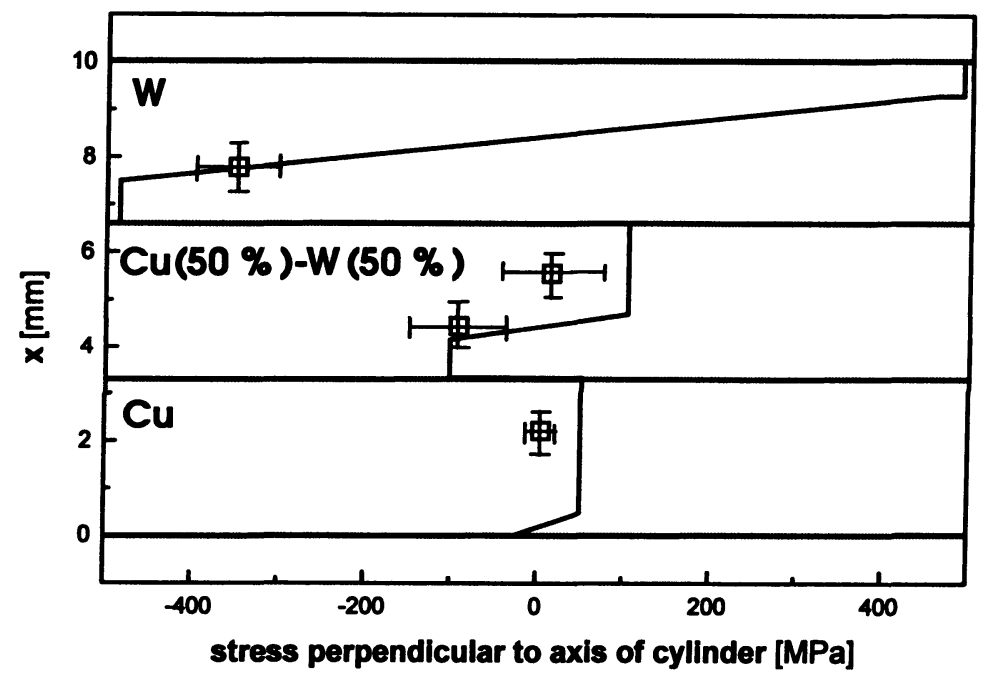

FIGURE 6 Comparing experimentally determined macrostresses against theoretical results.

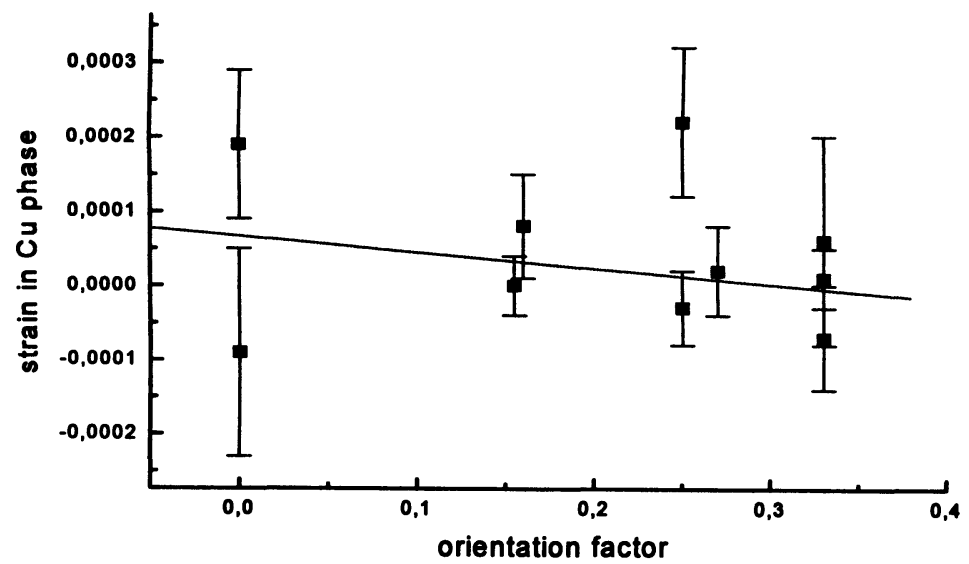

FIGURE 7 Single peak evaluation according to the elastic theory, the relative deformation should be linearly dependent on the orientation factor.

So far the results for the eight-layer sample cannot be compared with theoretical calculations. The experimentally determined stresses of both phases are plotted in Fig. 8(a) and (b). The most obvious two observations are firstly that the stress in the copper phase does barely exceed $100 \mathrm{MPa}$ whereas the tungsten reaches up to $500 \mathrm{MPa}$ and secondly that 
the stresses in copper are compressional. The first observation may have its reason in the different yield strength of the two materials which are 60 and $550 \mathrm{MPa}$ respectively and are exceeded only by a few copper data points and that only in compression. This again hints at plastical behaviour if one compares it with recent calculations by Schaller (1997) which confirm that the theoretical residual stresses are lowered to about the yield strength if plasticity is included into the model. The yield strength, however, has no fixed single value but is dependent on microstructural
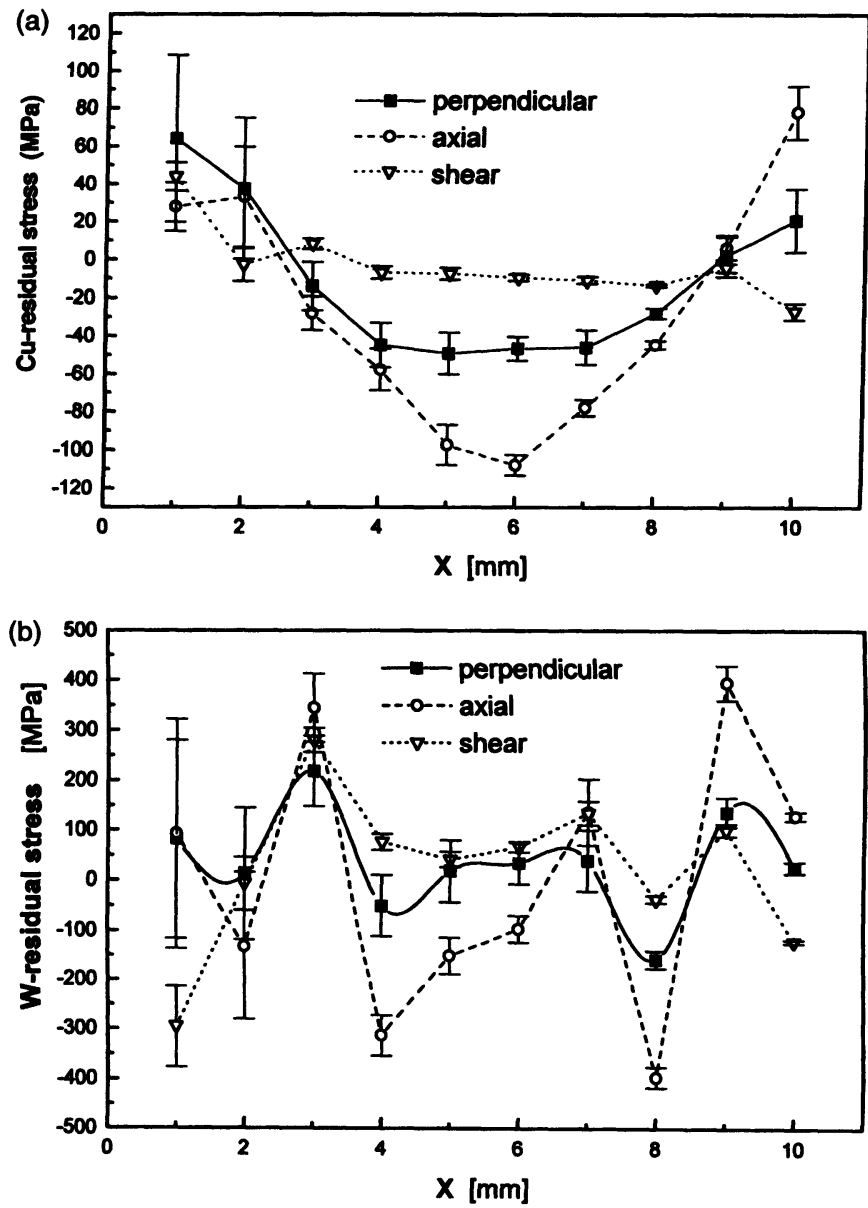

FIGURE 8 Residual stresses in (a) copper and (b) tungsten phase (eight-layer sample). 
parameters such as grain size, layer thickness and impurities. Consequently, the different yield strengths of copper and tungsten can explain the tendency in the maximum measured stress of the two phases, but quantitative statements ought to be evaluated very carefully.

The second observation seems at first glance to be contradictory to both theory and previous measurements. This disagreement can be resolved to a certain degree if one calculates the macrostresses $\left(\sigma_{\mathrm{m}}=x \sigma_{\mathrm{W}}+(1-x) \sigma_{\mathrm{Cu}}\right)$ from both phases and subtracts them from the
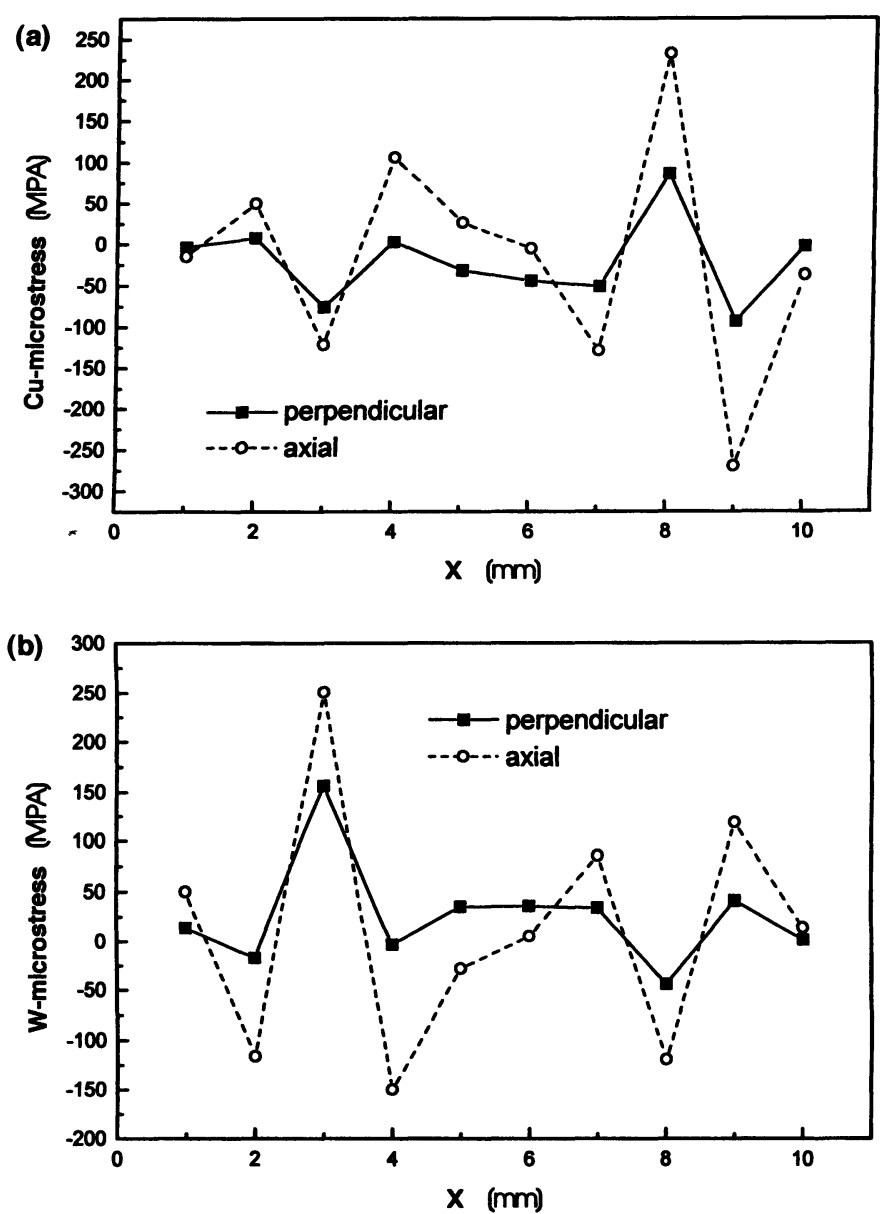

FIGURE 9 Microstresses in (a) copper and (b) tungsten phase (eight-layer sample). 
stresses of Fig. 8 thus obtaining the microstress distribution (Fig. 9). This displays that the actual residual load share of both phases is obviously much more complicated and may even depend on different parameters such as the macrostress.

\section{CONCLUSIONS}

The Neutron Fourier Diffractometer is a suitable tool to analyze residual macro- and microstresses in gradient materials. Different WCu samples were investigated. The phase fractions resulting from a Rietveld refinement are in good agreement with the ratio of the ingredient powders. According to their different yield strengths the maximum stress in tungsten and copper are quite different, namely 500 and 100 MPA respectively. Both experimental and theoretical results point to remarkable plastic deformation in the copper phase. The linear elastic theory is not sufficient and describes the experimental results only qualitatively.

\section{References}

Aksenov, V.L., Balagurov, A.M., Simkin, V.G., Taran, Yu.V., Kroening, M., Schreiber, J. et al., Utilization of High Resolution Fourier Diffractometer at IBR-2 reactor for strain measurement: first results and perspectives, 1st European Conference on Neutron Scattering, ECNS'96, Interlaken, Switzerland, 1996.

Fischer, In "grundlegende Untersuchungen zur Auslegung thermomechanisch extrem beanspruchter Gradientenwerkstoffe", Zwischenbericht, Saechsisches Staatsministerium fuer Wissenschaft und Kunst, Förderzeichen: 4-7531.50-03-EPW/602, 1996.

Hiismäki, P. et al., Exploitation of the Fourier Chopper in neutron diffractometry at pulsed sources. J. Appl. Crust, 21, 349-354. 1988.

Ilschner, B. and Cherradi, N., Proc. 3rd Int. Symp. on Functional Gradient Materials FGM'94, Presses polytechniques et universitaire romande, Lausanne, Switzerland, 1995.

Itoh, Y., Takahashi, M. and Takano, H., Design of tungsten/copper graded composite for high heat flux components, Fusion Engineering and Design, 31, 279-289, 1996.

Jedamzik, R., Neubrand, A. and Roedel, J., Electrochemical processing and characterization of graded tungsten/copper composites, Proc. 14th Intern. Plansee Seminar, Eds.: Kneringer et al., Vol. 1, p. 1, 1997.

Joensson, M., In "grundlegende Untersuchungen zur Auslegung thermomechanisch extrem beanspruchter Gradientenwerkstoffe", Zwischenbericht, Saechsisches Staatsministerium für Wissenschaft und Kunst, Förderzeichen: 4-7531.50-03EPW/602, 1996.

Pöyry, H., Hiismäki, P. and Virjo, A., Principles of RTOF Spectroscopy with Fourier Chopper Applications. Nucl. Instr. Methods, 126, 421-433, 1975.

Rabin, B.H. and Shiota, I., Functionally gradient materials, MRS Bulletin, Jan. 1995.

Schaller, W., Berechnung thermischer Spannungen in einem gradierten Wolfram-Kupfer Verbundwerkstoff, report for the Deutsche Forschungsgemeinschaft (DFG), Karlsruhe, 1997.

Young, R.A., The Rietveld Method, Oxford University Press, 1993. 